\title{
The Role of Hydroxychloroquine in COVID-19 Treatment: A Systematic Review and Meta-Analysis
}

Amir Shamshirian, ${ }^{1,2}{ }_{B S c}, P h D$, Amirhossein Hessami ${ }^{3,4}{ }_{M D}$, Keyvan Heydari, ${ }^{2,3}{ }_{M D}$, Reza Alizadeh-Navaei, ${ }^{2} M D, P h D$, Mohammad Ali Ebrahimzadeh, ${ }^{5}{ }_{\text {PharmD, PhD }}$, George W Yip, ${ }^{6}$ PhD, MBBS, Roya Ghasemian, ${ }^{7}{ }_{M D}$, Meghdad Sedaghat, ${ }^{8}{ }_{M D}$, Hananeh Baradaran, ${ }^{9}$ PharmD, PhD, Soheil Mohammadi Yazdi,${ }^{10}$ Elham Aboufazeli, ${ }^{11}$ PharmD, Hamed Jafarpour, ${ }^{3}{ }_{M D}$, Ehsan Dadgostar, ${ }^{12} M D$, Behnaz Tirandazi ${ }^{13}$ Reza Sadeghnezhad, ${ }^{14}{ }_{B S c}$, Keyvan Karimifar, ${ }^{15}$ MD, Aida Eftekhari,,${ }^{15}$ Danial Shamshirian, ${ }^{16}$ PharmD, PhD

\begin{abstract}
Objective: A systematic review and meta-analysis was carried out to examine the role of hydroxychloroquine (HCQ) in the treatment of COVID-19.

Methods: We performed a systematic search in PubMed, Scopus, Embase, CochraneLibrary, Web of Science, Google Scholar, and medRxiv pre-print databases using available MeSH terms for COVID-19 and hydroxychloroquine. Data from all studies that focused on the effectiveness of HCQ with or without the addition of azithromycin (AZM) in confirmed COVID-19 patients, which were published up to 12 September 2020, were collated for analysis using CMA v.2.2.064.

Results: Our systematic review retrieved 41 studies. Among these, 37 studies including 45,913 participants fulfilled the criteria for subsequent meta-analysis. The data showed no significant difference in treatment efficacy between the HCQ and control groups (RR: $1.02,95 \%$ CI, 0.81-1.27). Combination of HCQ with AZM also did not lead to improved treatment outcomes (RR: $1.26,95 \%$ CI, 0.91-1.74). Furthermore, the mortality difference was not significant, neither in HCQ treatment group (RR: 0.86, 95\% CI, 0.71-1.03) nor in HCQ plus AZM treatment group (RR: $1.28,95 \%$ CI, $0.76-2.14$ ) in comparison to controls. Meta-regression analysis showed that age was the factor that significantly affected mortality $(P<0.00001)$.

Conclusion: The meta-analysis found that there was no clinical benefit of using either HCQ by itself or in combination with AZM for the treatment of COVID-19 patients. Hence, it may be prudent for clinicians and researchers to focus on other therapeutic options that may show greater promise in this disease.
\end{abstract}

Ann Acad Med Singap 2020;49:789-800

Keywords: Azithromycin, coronavirus outbreaks, pandemic, SARS-CoV-2 disease

\footnotetext{
${ }^{1}$ Department of Medical Laboratory Sciences, Student Research Committee, School of Allied Medical Science, Mazandaran University of Medical Sciences, Sari, Iran

${ }^{2}$ Gastrointestinal Cancer Research Center, Non-Communicable Diseases Institute, Mazandaran University of Medical Sciences, Sari, Iran

${ }^{3}$ Student Research Committee, School of Medicine, Mazandaran University of Medical Sciences, Sari, Iran

${ }^{4}$ Systematic Review and Meta-Analysis Expert Group (SRMEG), Universal Scientific Education and Research Network (USERN), Tehran, Iran

${ }^{5}$ Pharmaceutical Sciences Research Center, Department of Medicinal Chemistry, School of Pharmacy, Mazandaran University of Medical Science, Sari, Iran

${ }^{6}$ Department of Anatomy, Yong Loo Lin School of Medicine, National University of Singapore, Singapore

${ }^{7}$ Antimicrobial Resistance Research Center, Department of Infectious Diseases, Mazandaran University of Medical Sciences, Sari, Iran

${ }^{8}$ Department of Internal Medicine, Imam Hossein Hospital, Shahid Beheshti University of Medical Sciences, Tehran, Iran

${ }^{9}$ Clinical Pharmacy Department, Faculty of Pharmacy, Tehran University of Medical Sciences, Tehran, Iran

${ }^{10}$ Student Research Committee, Kashan University of Medical Sciences, Kashan, Iran

${ }^{11}$ Department of Research and Development, IRAN NAJO Pharmaceutical Company, Tehran, Iran

${ }^{12}$ Department of Psychiatry, School of Medicine, Isfahan University of Medical Sciences, Isfahan, Iran

${ }^{13}$ Department of Physical Medicine and Rehabilitation, Neuromusculoskeletal Research Center, Iran University of Medical Sciences, Tehran, Iran

${ }^{14}$ Student Research Committee, Faculty of Health, Health Sciences Research Center, Mazandaran University of Medical Sciences, Sari, Iran

${ }^{15}$ Student Research Committee, School of Medicine, Mashhad University of Medical Sciences, Mashhad, Iran

${ }^{16}$ Chronic Respiratory Diseases Research Center, National Research Institute of Tuberculosis and Lung Diseases (NRITLD), Shahid Beheshti University of Medical Sciences, Tehran, Iran

Addresses for Correspondence: Dr Danial Shamshirian, Chronic Respiratory Diseases Research Center, National Research Institute of Tuberculosis and Lung Diseases (NRITLD), Shahid Beheshti University of Medical Sciences, Tehran, Iran. Email: shamshirian@sbmu.ac.ir

Dr Reza Alizadeh-Navaei; Gastrointestinal Cancer Research Center, Mazandaran University of Medical Sciences, Sari, Iran. Email: alizadeh@mazums.ac.ir
} 


\section{Introduction}

The World Health Organization (WHO) declared COVID-19 as a pandemic disease on 26 March 2020. ${ }^{1,2}$ By 12 September 2020, the WHO COVID-19 dashboard reported that $28,329,790$ people had been afflicted by COVID-19 worldwide, with a total of 911,877 deaths. There are still no officially approved therapeutic measures against COVID-19 and to date, WHO's fundamental advice to the public for prevention of this disease is the promotion of good personal hygiene, observance of social distancing, and quarantine of infectious cases. ${ }^{3}$

In the case of therapeutics, there are several candidate drug and non-drug treatment types classified by WHO. ${ }^{4}$ Also, according to the Coronavirus Treatment Acceleration Program (CTAP) of the US Food and Drug Administration (FDA), as of 31 August 2020, there were approximately 590 drug development programmes, 310 trials and 5 authorised treatments only for emergency use. However, there is still no FDA-approved treatment specifically for COVID-19. ${ }^{5}$

Hydroxychloroquine (HCQ), used either alone or in combination with azithromycin (AZM), is one of numerous controversial therapies for COVID-19 patients that are being actively investigated. While some studies have shown promising results from the use of HCQ in preventing or treating COVID-19 infections, ${ }^{6-8}$ other authors have reported that this drug produced no significant beneficial effects, and may even lead to harmful outcomes for patients. ${ }^{9-11}$ The controversy has ignited heated debates not just within the scientific and medical fraternity, but in political circles as well. ${ }^{12,13}$ This systematic review and meta-analysis aims to address this, and to provide a clearer understanding of the effectiveness of HCQ in the treatment of COVID-19.

\section{Method}

\section{Search strategy}

The Preferred Reporting Items for Systematic Reviews and Meta-Analyses (PRISMA) guideline was used for study design, search protocol, screening and reporting. A systematic search was performed using PubMed, Scopus, Embase, Cochrane Library, Web of Science and Google Scholar, as well as the pre-print database of medRxiv, to retrieve all published studies up to 12 September 2020. Additional data was extracted from gray literature and cited references of published papers. The search strategy included all MeSH terms and free keywords on COVID-19, SARS-CoV-2 and hydroxychloroquine(Table 1). The search did not impose any restriction on the date, geographical location or language of the published studies.

Table 1. Search strategy terms

\begin{tabular}{|c|c|c|c|}
\hline PICO & Keywords & \#* & Search Terms \\
\hline 离 & COVID-19 & 1 & $\begin{array}{l}\text { "COVID-19" OR "2019 novel coronavirus disease" OR "COVID19" OR "COVID-19 pandemic" } \\
\text { OR "SARS-CoV-2 infection" OR "COVID-19 virus disease" OR "2019 novel coronavirus } \\
\text { infection" OR "2019-nCoV infection" OR "2019-nCoV" OR "coronavirus disease } 2019 " \text { OR } \\
\text { "coronavirus disease-19" OR "2019-nCoV disease" OR "COVID-19 virus infection" OR "severe } \\
\text { acute respiratory syndrome coronavirus 2" OR "Wuhan coronavirus" OR "SARS-CoV-2" OR } \\
\text { "2019 novel coronavirus" OR "COVID-19 virus" OR "coronavirus disease } 2019 \text { virus" OR } \\
\text { "COVID19 virus" OR "Wuhan seafood market pneumonia virus" }\end{array}$ \\
\hline
\end{tabular}

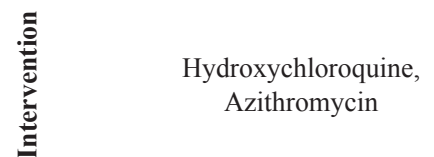

อี
2 "Hydroxychloroquine" OR "Oxychlorochin" OR "Oxychloroquine" OR "Hydroxychlorochin" OR "Plaquenil" OR "Hydroxychloroquine Sulfate" OR "Hydroxychloroquine Sulfate (1:1) Salt"

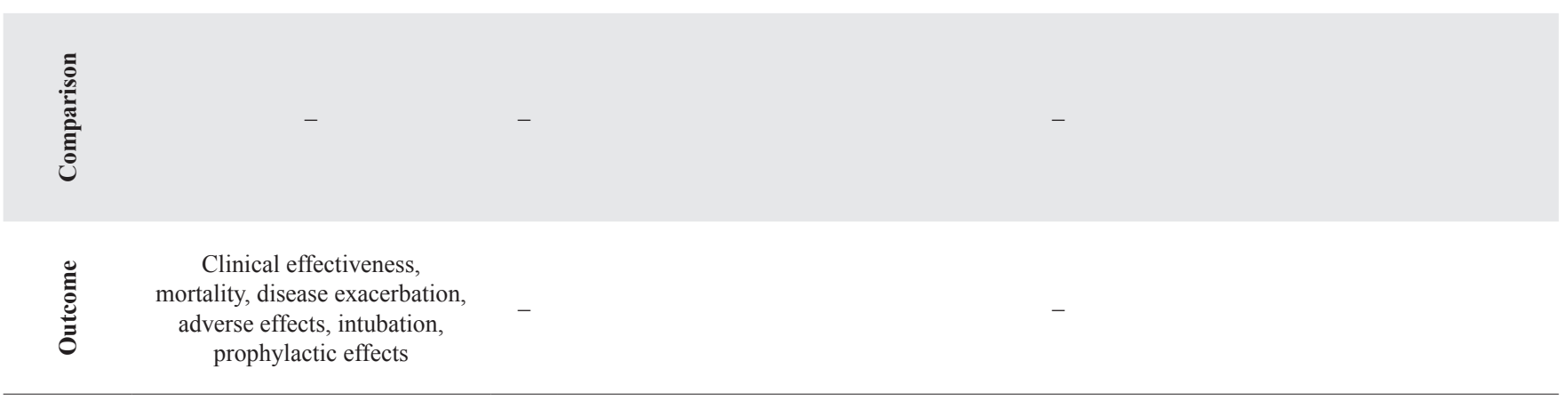

* \#1 and \#2 combined with "AND" operator

$\checkmark$ To widen search results and avoid missing data, terms for azithromycin, comparison and outcomes were not included in the search strategy. 


\section{Criteria for study selection}

Two researchers in the team performed screening and selection of the papers independently. A third party of the team served as the arbitrator for all disagreements. Studies that met the following criteria were included in the meta-analysis: (1) comparative or non-comparative clinical studies, including observational/interventional studies of a retrospective/prospective nature with/without control group as well as Randomised Clinical Trials (RCTs); or (2) studies that reported the effect of HCQ with/ without AZM in confirmed cases of COVID-19. Papers were excluded if they were: (1) reports on in vitro or animal studies; (2) reviews; (3) case reports; (4) duplicate publications; or (5) lacking sufficient information for calculation of desired parameters.

\section{Data extraction \& quality assessment}

Two researchers in the team performed quality assessment of the studies and extracted data from the selected papers independently. A third team member resolved any disagreements in this step. The data extraction checklist included the name of the first author, publication year, region of study, number of patients, number of controls, mean age, treatment option, medication dosage, treatment duration, adverse effects and nasopharyngeal culture status through Reverse Transcription-Polymerase Chain Reaction (RT-PCR) and mortality.

The Jadad scale, ROBINS-I tool and Newcastle-Ottawa Scale (NOS) checklists were used to evaluate the selected randomised controlled trials, non-randomised controlled trials and observational studies, respectively, based on multiple aspects of the study methodology and study process. Risk-of-bias plots were created using the robvis online tool. ${ }^{14}$

\section{Targeted outcomes}

Targeted outcomes included: (1) clinical effectiveness of HCQ with/without AZM in the treatment of COVID-19; (2) mortality rates; (3) disease exacerbation; (4) frequency of known HCQ adverse effects occurring during treatment; (5) need for intubation; and (6) prophylactic effects of HCQ.

The following were performed: (1) HCQ compared to a control group that was given standard treatment; and (2) HCQ plus AZM compared to a control group that was given standard treatment.

These definitions were used to assess the outcomes:

Clinical effectiveness: nasopharyngeal swab with a negative result by RT-PCR test.
Disease exacerbation: clinical symptoms of the disease were worsened.

Adverse effects: occurrence of symptoms known to be related to HCQ, such as diarrhoea, vomiting, blurred vision, rash, headache, etc.

Group A in forest plots: case groups that received HCQ with/without the AZM regimen.

Group B in forest plots: control groups without $\mathrm{HCQ} /$ HCQ plus AZM regimen.

\section{Heterogeneity assessment}

I-square $\left(\mathrm{I}^{2}\right)$ statistic was used for heterogeneity evaluation. Following the Cochrane Handbook for Systematic Reviews of Interventions, ${ }^{15}$ the $\mathrm{I}^{2}$ was interpreted as follows: " $0 \%$ to $40 \%$ : might not be important; $30 \%$ to $60 \%$ : may represent moderate heterogeneity; $50 \%$ to $90 \%$ : may represent substantial heterogeneity; $75 \%$ to $100 \%$ : considerable heterogeneity. The importance of the observed value of $\mathrm{I}^{2}$ depends on (i) magnitude and direction of effects and (ii) strength of evidence for heterogeneity (e.g. $P$-value from the chisquare test, or a confidence interval for $\left.I^{2}\right)$."

In cases where heterogeneity was present, the DerSimonian and Laird random-effects model was applied to pool the outcomes; otherwise, the inverse variance fixed-effect model was used. Forest plots were used to visualise the degree of variation among studies.

\section{Data analysis}

Statistical analysis was performed using the Comprehensive Meta-Analysis (CMA) v. 2.2.064 software. Risk Ratio (RR) or Odds Ratio (OR) were used for outcome estimation, whenever appropriate, with 95\% Confident Interval (CI). The fixed/random-effects models were used based on the heterogeneity status. In the case of zero frequency, a correction value of 0.1 was used. Meta-regression analysis was performed to examine the impact of patient age on HCQ regimen group mortality RR. However, due to unavailability of data, we could not apply meta-regression analysis on the other potential moderator variables such as sex, underlying disease, etc.

\section{Publication bias and sensitivity analysis}

Begg's and Egger's tests, as well as the funnel plot, were used for publication bias evaluation. A $P$-value of less than 0.05 was considered to be statistically significant. Additionally, we conducted a sensitivity analysis to examine the effect of studies that greatly influenced the results, especially by their weight, by excluding them from the meta-analysis. ${ }^{16}$ 


\section{Results}

\section{Study selection process}

The database search found 4,358 papers. After exclusion of duplicated papers and the initial screening, 236 papers were assessed for eligibility. Thirty-nine papers were used for qualitative synthesis, with meta-analysis performed on 37 of them. The PRISMA flow diagram of the study selection process is presented in Fig. 1.

\section{Study characteristics}

The HCQ arm of comparative studies was combined with observational studies for effect size meta-analysis of the 37 publications. The sample size of the studies ranged from 11 to 8,075, with a total of 45,913 cases. The characteristics of the studies that entered into the systematic review are shown in Table 2.

\section{Quality assessment}

Quality assessments of studies entered into the metaanalysis performed using the Jadad, ROBINS- $I$ and
NOS checklists are reported in Table 2. The risk of bias summary is shown in Fig. 2.

\section{Publication bias}

The Begg's and Egger's tests for every performed analysis gave insignificant results: HCQ regimen effectiveness $\left(P_{B}=0.60 ; P_{E}=0.29\right)$; association between HCQ $\left(P_{B}=0.71 ; P_{E}=0.41\right)$ and HCQ plus AZM $\left(P_{B}=0.25 ; P_{E}=0.78\right)$ regimen and mortality rate in controlled randomised and non-randomised studies. However, a moderate publication bias was observed regarding overall mortality in all the studies $\left(P_{B}=0.54\right.$; $\left.P_{E}=0.02\right)$.

\section{Meta-Analysis Findings}

\section{Treatment outcome}

\section{Hydroxychloroquine regimen effectiveness}

The meta-analysis of risk ratios for HCQ effectiveness in all the comparative randomised and non-randomised studies (Fig. 3) found no significant difference between

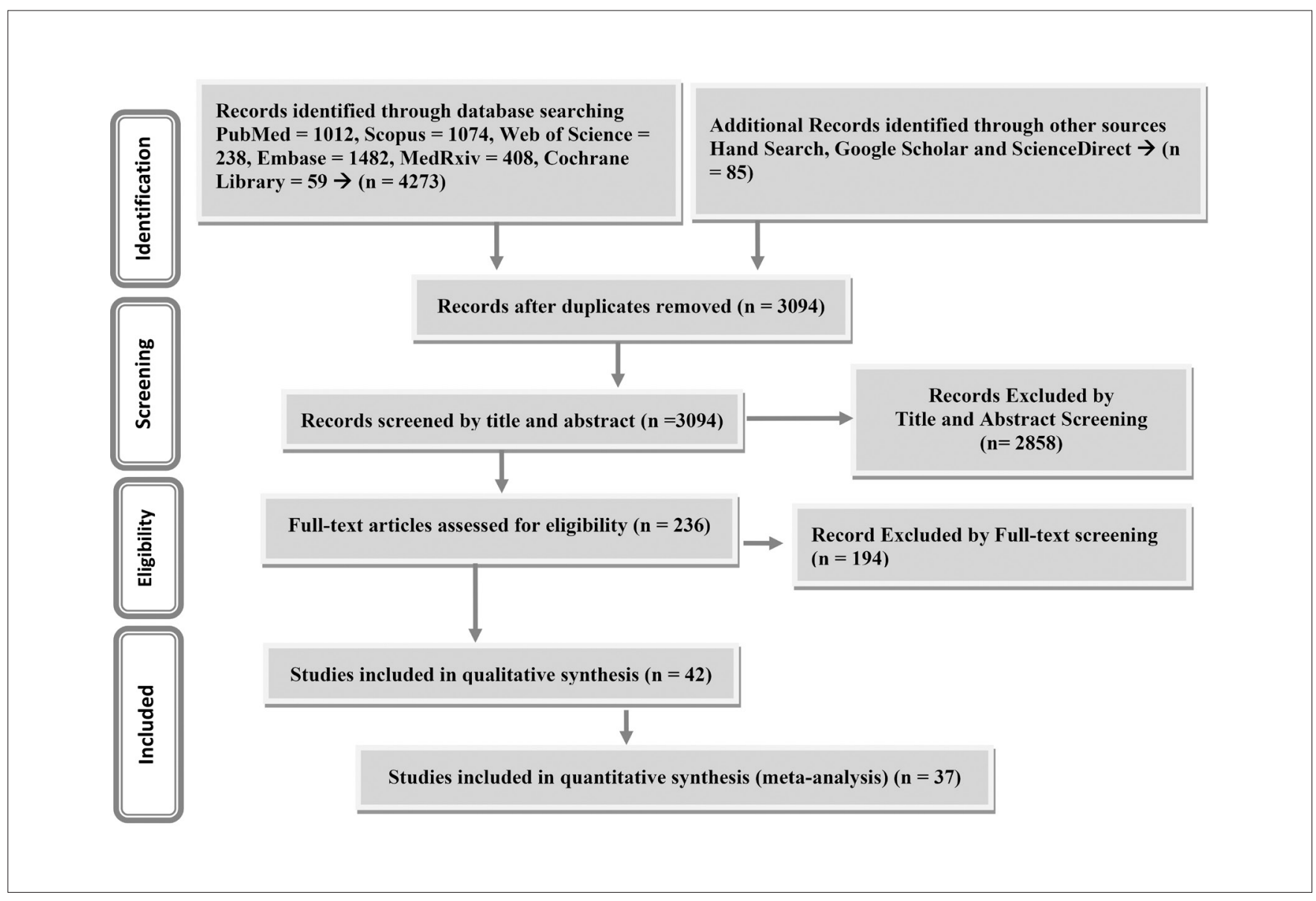

Fig. 1. PRISMA flow diagram of the study selection process 


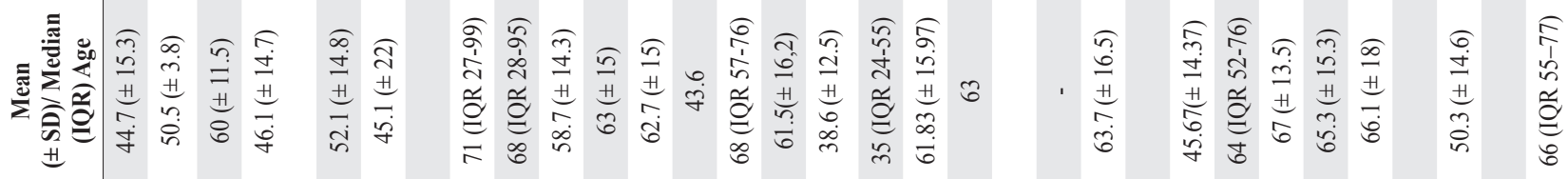

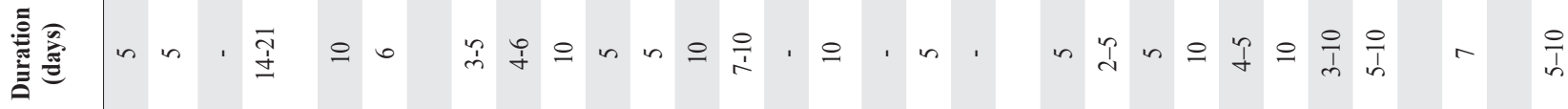

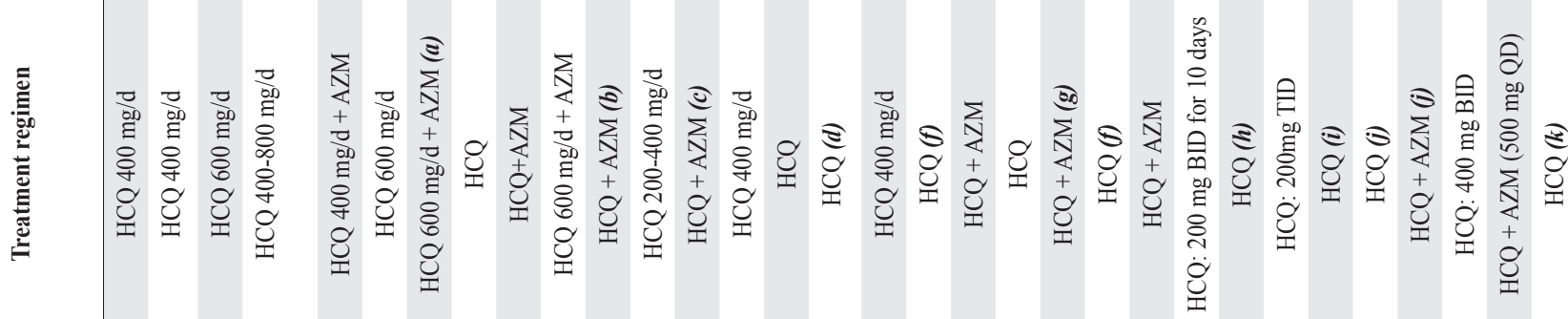

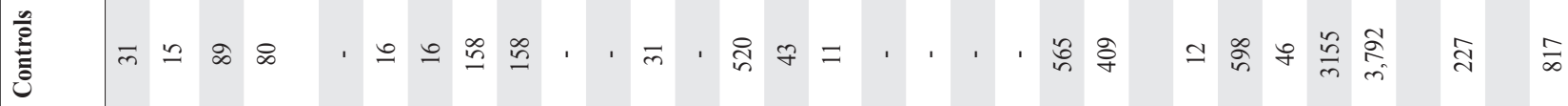

שّ̋

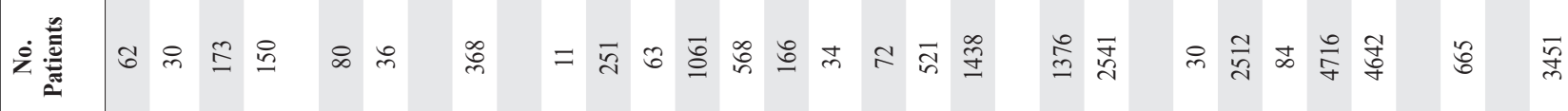

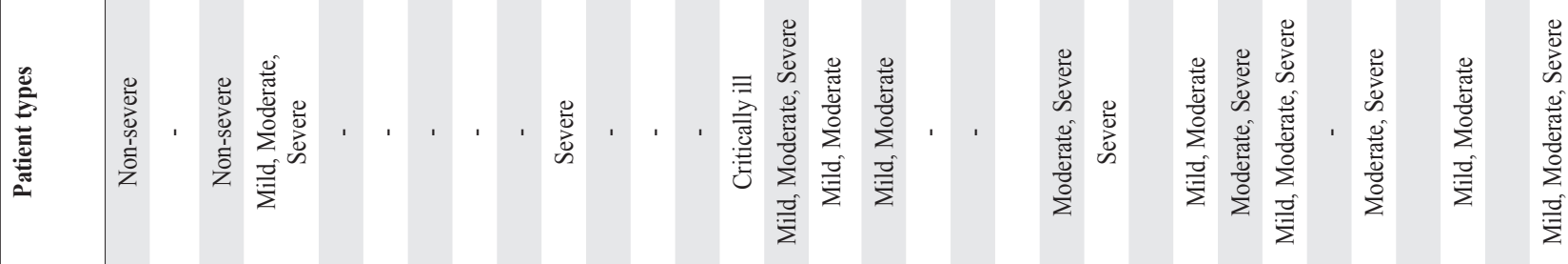

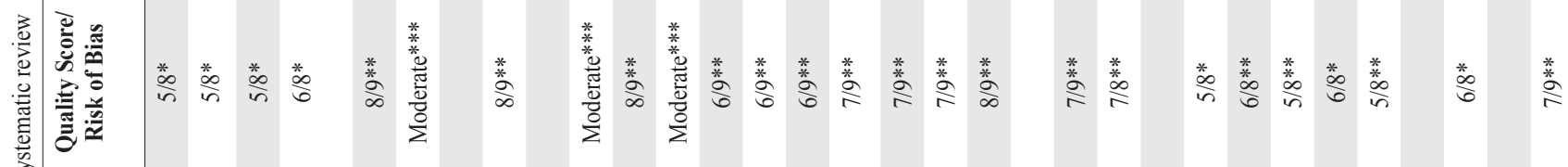

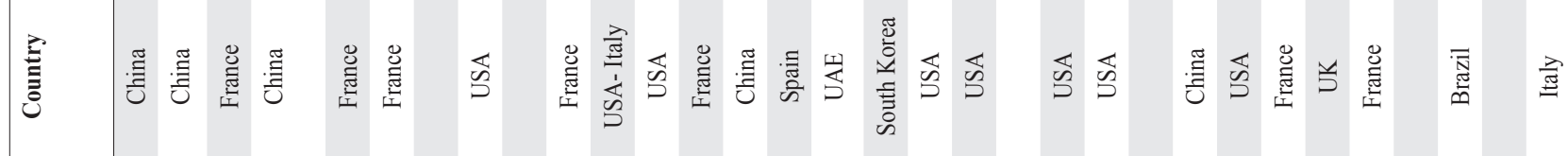

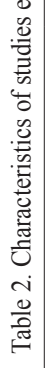

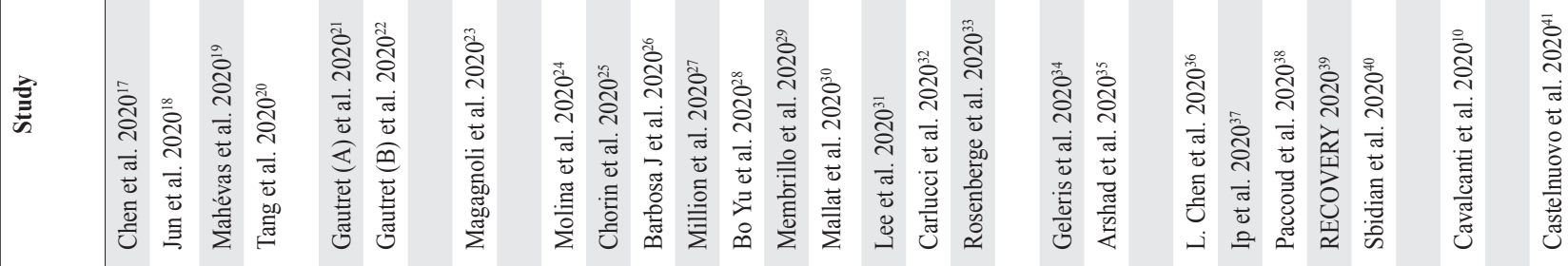




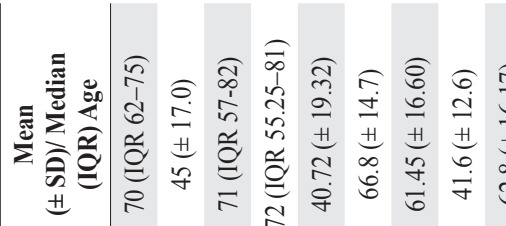

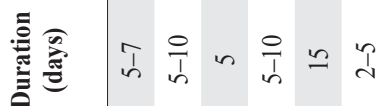

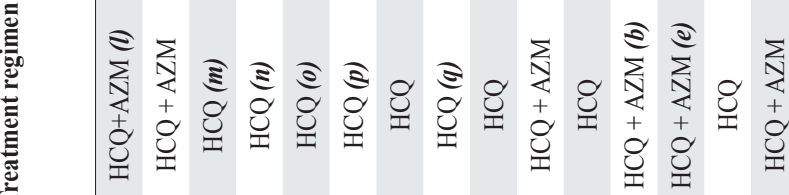

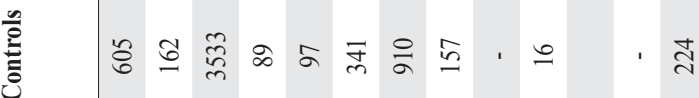

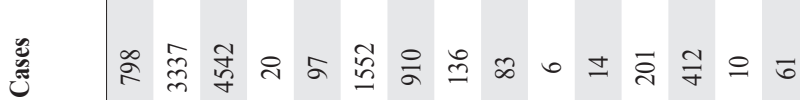

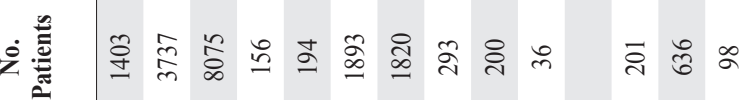

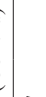

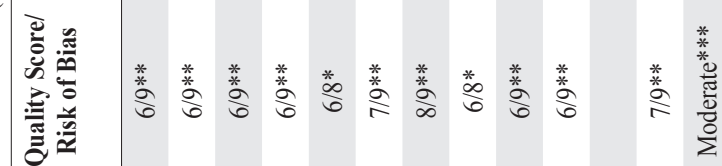

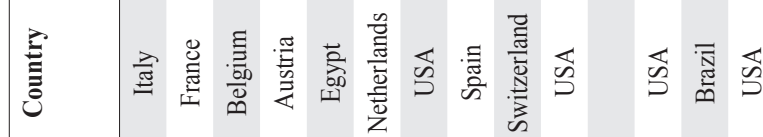

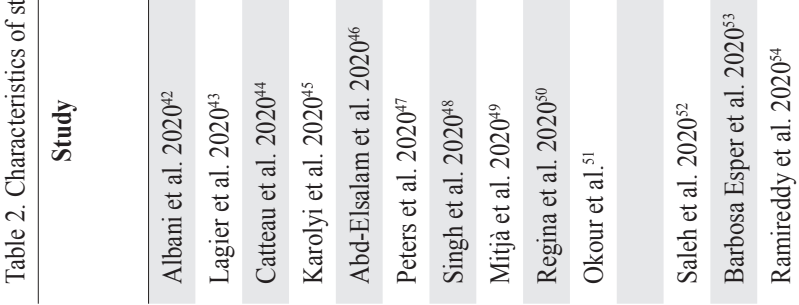

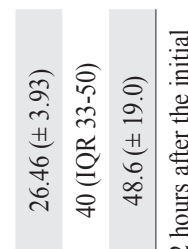

$$
\text { : }
$$

芒

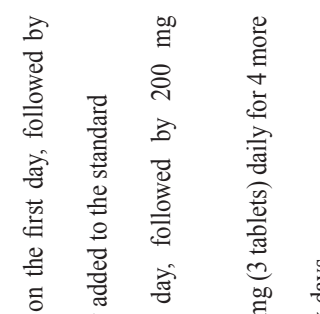

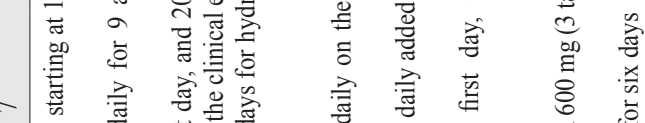

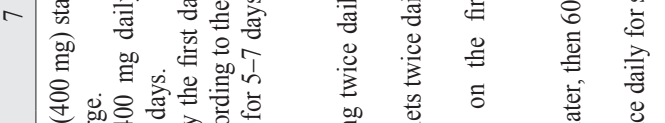

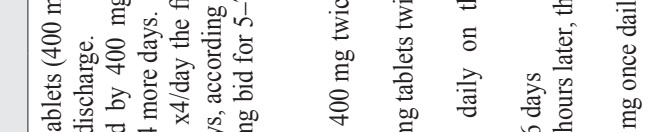

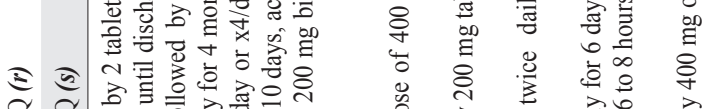

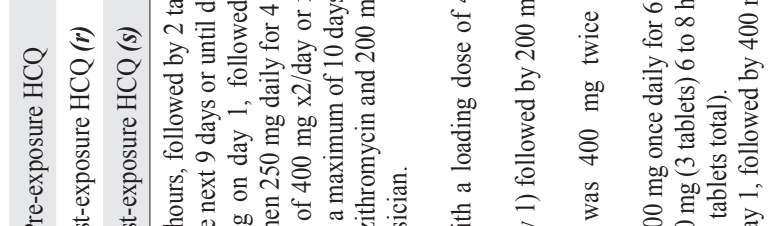

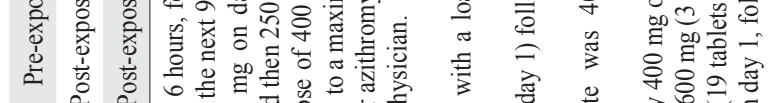

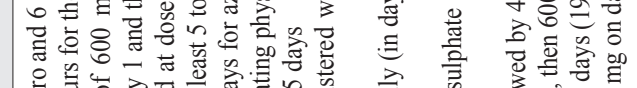

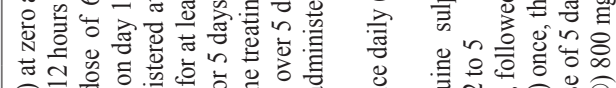

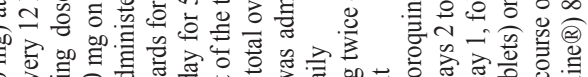

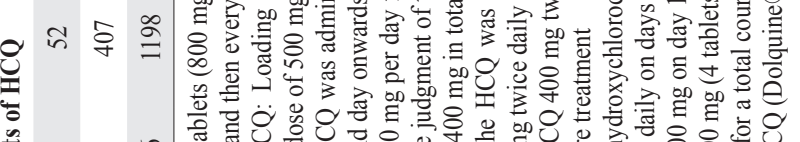

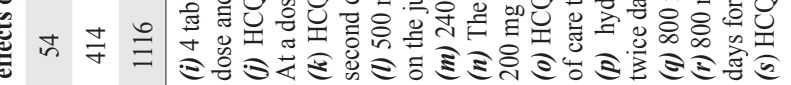

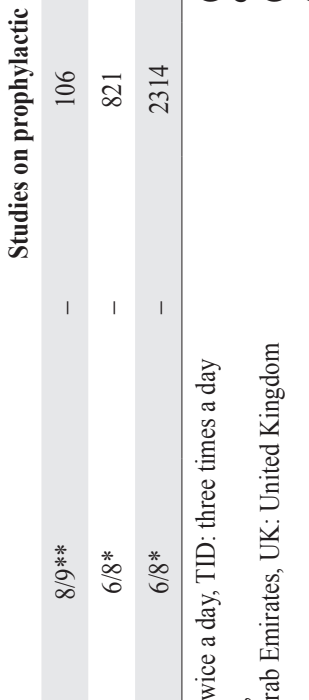

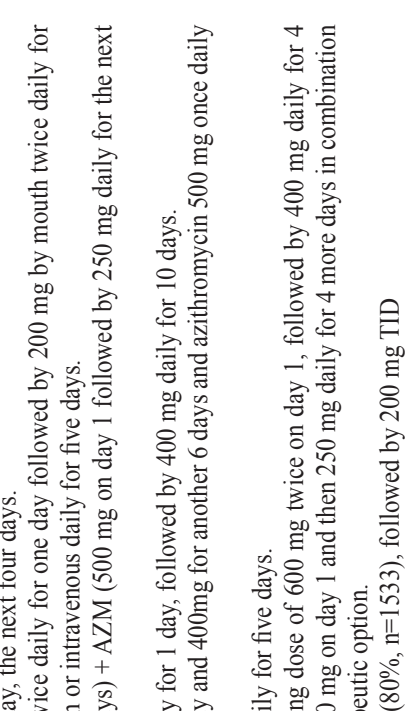

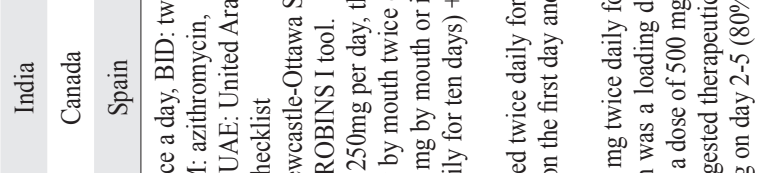

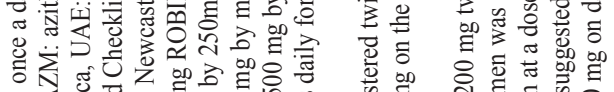

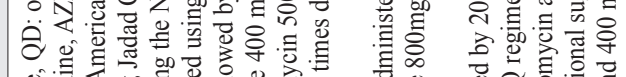

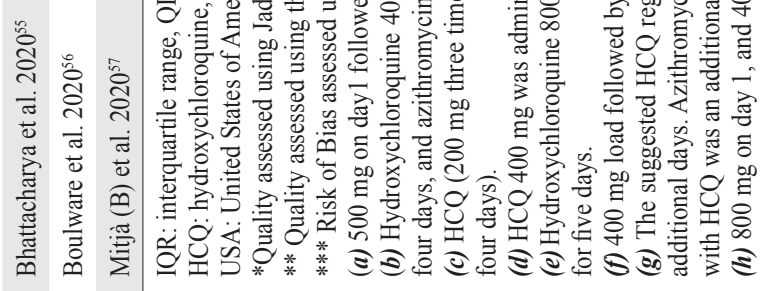


Random sequence generation Allocation concealment Blinding of participants and personnel Blinding of outcome assessment Incomplete outcome data Selective reporting Other sources of bias Overall

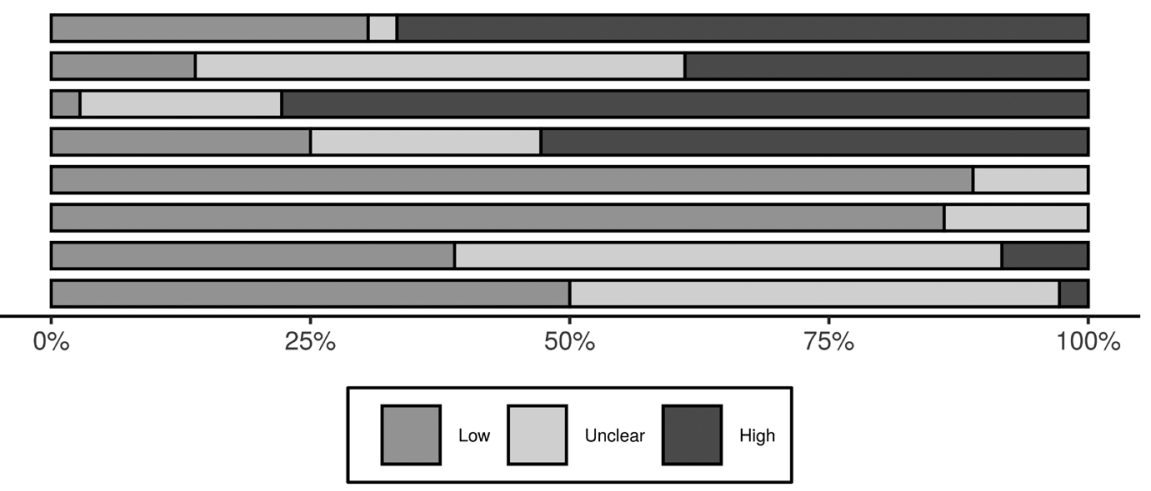

Fig. 2. Summary of risk of bias for studies entered into the meta-analysis

the case group (standard treatment with HCQ regimen) and the control group (standard treatment without HCQ; RR: $1.02,95 \%$ CI, 0.81-1.27; RD: 0.01, 95\% CI, $-0.12-$ $0.15)$. Meta-analysis of controlled randomised studies showed no substantial effectiveness of HCQ (RR: 1.19, 95\% CI, 0.87-1.63; RD: 0.12, 95\% CI, -0.07-0.33).
Sensitivity analysis for hydroxychloroquine regimen effectiveness

To evaluate the impact of inverse RRs as well as the weight of different studies on the meta-analysis results, we conducted several sensitivity analyses. (1) Despite

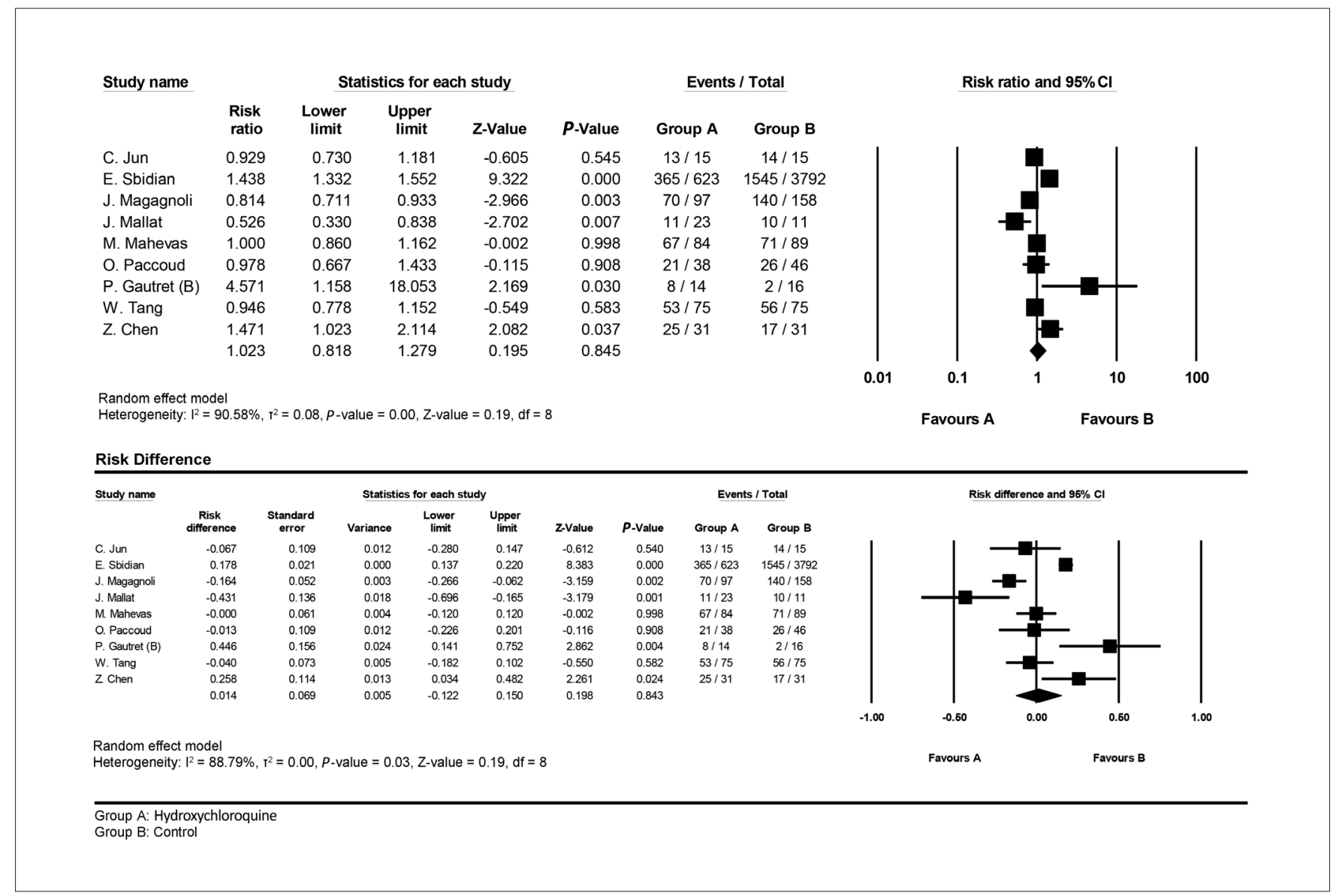

Fig. 3. Forest plot for pooling risk ratios and risk differences regarding hydroxychloroquine regimen in comparative randomised and non-randomised studies 
the substantial relative weight of the Sbidian et al. study, exclusion of this study from the meta-analysis did not significantly change the results (RR: $0.94,95 \% \mathrm{CI}$, $0.80-1.11$ ). (2) Of the 5 studies that reported $P$-values of less than $0.05,3$ have a $P$ value less than 0.05 in favour of Group A and 2 have a $P$-value below 0.05 in favour of Group B. These are the Magagnoli et al. and Mallat et al. studies, in which the $95 \%$ CI of the RR does not intersect with that from the Chen et al., Gautret (B) et al. and Sbidian et al. reports. Excluding the papers by Magagnoli et al. and Mallat et al. from the sensitivity analysis did not have any effect (RR: $1.14,95 \%$ CI, 0.92-1.41). (3) Exclusion of these studies showed no significant difference in the meta-analysis (RR: 0.89, 95\% CI, 0.78-1.00). (4) To maximise the analysis validity, exclusion of pre-prints data from meta-analysis did not significantly change the results (RR: $0.93,95 \%$ CI, 0.82-1.06).

\section{Hydroxychloroquine plus azithromycin regimen}

No significant difference was found in the effectiveness of the HCQ plus AZM combination regimen compared to the control group in the meta-analysis (RR: $1.26,95 \%$ CI, 0.91-1.74). A considerable risk difference was present between the groups (RD: $0.28,95 \% \mathrm{CI}, 0.01-0.54$ ). Also, by excluding pre-prints data from meta-analysis, sensitivity analysis showed no significant differences for HCQ plus AZM regimen (RR: 2.28, 95\% CI, 0.37-13.79).

\section{Hydroxychloroquine regimen and mortality rate}

Meta-analysis of comparative randomised and nonrandomised studies showed no significant difference in mortality rates between the HCQ regimen group and standard treatment group (RR:0.86, $95 \% \mathrm{CI}, 0.71-1.03$; RD: $-0.02,95 \% \mathrm{CI},-0.04-0.00)$. The sensitivity analysis found no significant difference in the mortality rate in the HCQ regimen arm compared to the control group by excluding pre-prints data (RR: 0.86, 95\% CI, 0.67-1.10).

\section{Meta-regression analysis of the effect of age on mortality}

Meta-regression showed that the age of patients had a significant effect on risk ratios with regard to mortality rate in the HCQ regimen group $(P<0.00001)$.

\section{Hydroxychloroquine plus azithromycin regimen and mortality rate}

Meta-analysis of mortality rates in comparative randomised and non-randomised studies found no significant difference in the HCQ plus AZM regimen group compared to the control group (RR: $1.28,95 \%$ CI, 0.76-2.14; RD: 0.09, 95\% CI, -0.02-0.20). Also, the sensitivity analysis result was not significant after excluding pre-prints (RR: $1.28,95 \%$ CI, 0.59-2.79).

\section{Overall mortality}

In the analysis of overall mortality, we considered the treatment arms of all comparative studies as observational studies. The pooled overall mortality rate was found to be $15.5 \%$ (95\% CI, $13.2 \%-18.0 \%$ ) for HCQ and $9.5 \%$ (95\% CI, 5.2\%-16.8\%) HCQ plus AZM regimen (Fig. 4). By excluding pre-prints from meta-analysis, the results did not change substantially.

\section{Disease exacerbation}

Meta-analysis of all comparative studies showed that disease exacerbation was not significantly different between the HCQ group and the control group (RR: $1.41,95 \%$ CI, 0.82-2.44; RD: 0.03, 95\% CI, -0.03-0.11). Exclusion of pre-prints data from meta-analysis did not significantly change the results (RR: $1.50,95 \% \mathrm{CI}$, 0.84-2.67). Meta-analysis of controlled randomised studies found no difference in disease exacerbation between two groups (RR: $0.62,95 \% \mathrm{CI}, 0.20-1.96$; RD: $-0.04,95 \%$ CI, $-0.13-0.05)$.

\section{Intubation}

Meta-analysis of comparative randomised and nonrandomised studies found no significant difference between the HCQ group and the control group in the odds of intubation during treatment (OR: $2.06,95 \% \mathrm{CI}$, $0.31-13.52)$.

\section{Adverse effects}

Meta-analysis of comparative randomised and nonrandomised studies showed that the odds of adverse effects in patients who received the HCQ regimen was approximately 3.5 times higher than the control group without HCQ regimen (OR: 3.40, 95\% CI, 1.65-6.98). Meta-analysis of controlled randomised studies found 4 times higher odds of experiencing adverse effects in patients who received the HCQ regimen compared to the control group (OR: 4.08, 95\% CI, 1.84-9.04). Exclusion of pre-prints from meta-analysis resulted in approximately 3 times higher chance of adverse effects (OR: 3.03, 95\% CI, 1.34-6.86).

\section{Meta-analysis of observational studies}

We considered the treatment arms of comparative studies as observational studies in this section. Meta-analysis showed that $26.8 \%$ of patients suffered from known HCQ adverse effects (95\% CI, $16.3 \%-40.7 \%)$; $65.3 \%$ (95\% CI, $56.7 \%-73.1 \%)$ of patients were discharged 


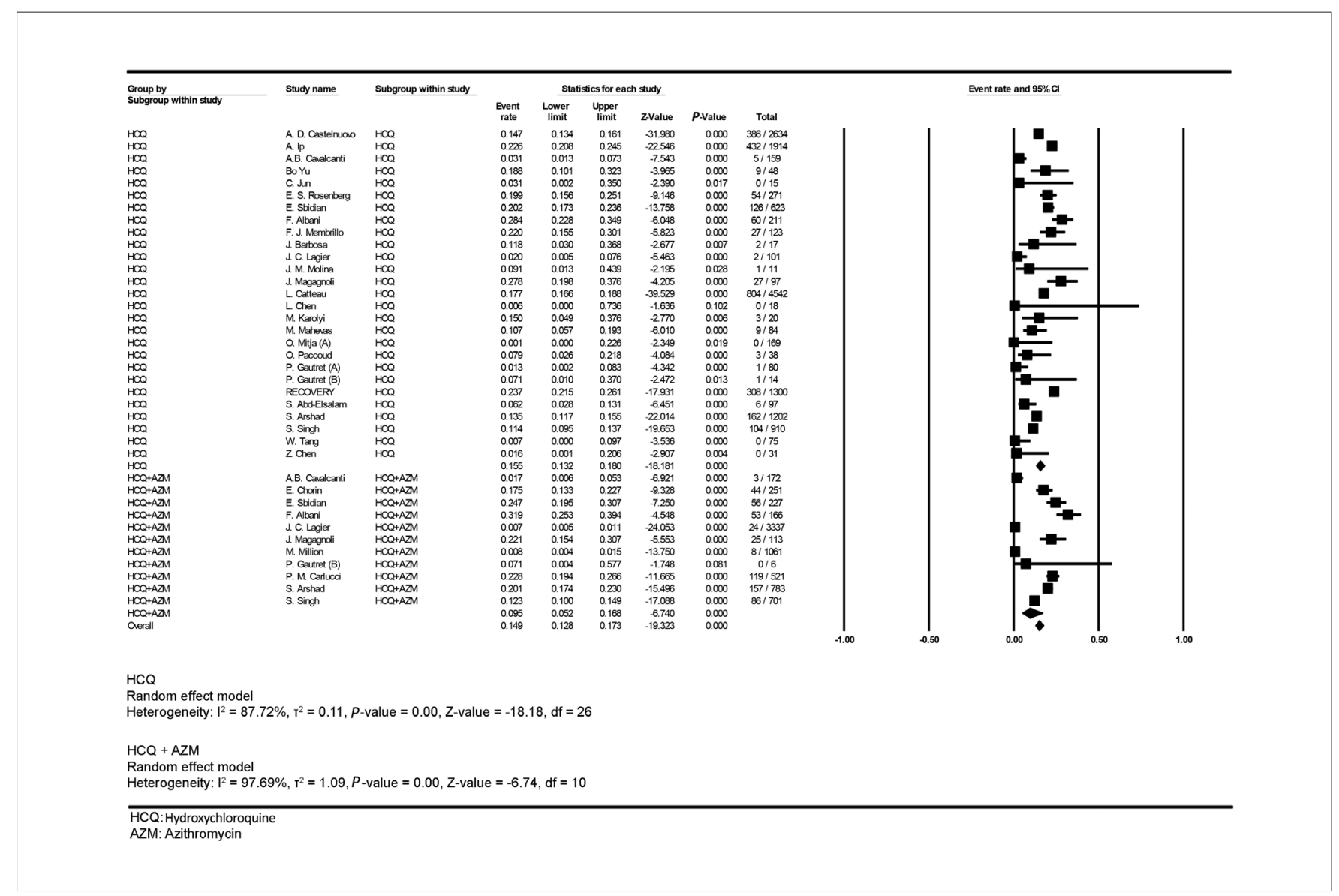

Fig. 4. Forest plot for pooling mortality rates

from hospitals or had negative RT-PCR results from their nasopharyngeal culture. In contrast, 23.3\% (95\% CI, $8.9 \%-48.6 \%)$ of patients suffered exacerbated disease, with $7.1 \%(95 \% \mathrm{CI}, 2.8 \%-17.0 \%)$ being admitted to the intensive care unit (ICU) and 23.8\% ( $95 \%$ CI, 6.6\%-57.9\%) undergoing intubation.

\section{Prophylactic effects of hydroxychloroquine}

Meta-analysis revealed no significant prophylactic effect of HCQ (OR: 0.58, 95\% CI, 0.20-1.66).

\section{Discussion}

The natural course of COVID-19 is such that more than $90 \%$ of patients will recover spontaneously from the infection. However, in a small proportion of cases, the disease progresses and leads to the development of Acute Respiratory Distress Syndrome and multi-organ failure ${ }^{58}$ Recent reports suggest that this progression may be due to cytokine storm, in which there is an uncontrolled release of pro-inflammatory cytokines into the plasma of patients. Thus, there is a critical need to identify anti-inflammatory agents to reduce the production and release of cytokines and pro-inflammatory factors. ${ }^{59}$

From as early as the 1950s, HCQ has been known to be an effective anti-inflammatory drug that is especially useful for the treatment of autoimmune disorders. ${ }^{60} \mathrm{~A}$ recent report by Yao et al. showed that HCQ may play an inhibitory role in SARS-CoV-2 infection in vitro. ${ }^{61}$ Pagliano et al. suggested that HCQ may be used as a pre/ post-exposure prophylaxis agent against SARS-CoV-2 infection for healthcare workers who were exposed to the virus in a contaminated environment. ${ }^{62}$

In contrast, Guastalegname and Vallone urged caution as the usefulness and potential harmful effects of HCQ in COVID-19 were not clear, and pointed out that treatment of Chikungunya viral infection with chloroquine led to dire paradoxical consequences., ${ }^{9,63}$ A similar cautionary opinion was also expressed by Kim et. al. ${ }^{64}$ Molina et al. followed up on 11 COVID-19 patients who were treated with an HCQ and azithromycin regimen, and found no clinical benefit or anti-viral activity. ${ }^{24}$ The pre-print of a quasi-randomised comparative study showed that 
HCQ not only did not provide any benefits to patients with COVID-19 but also increased the need for urgent respiratory support $(P=0.013){ }^{26}$ Similarly, Magagnoli et al. found that HCQ/HCQ plus AZM regimens failed to provide any clinical benefits to COVID-19 patients. ${ }^{23}$ Instead, patients in the HCQ group had a higher mortality rate (hazard ratio: $2.61,95 \% \mathrm{CI}, 1.10-6.17 ; P=0.03$ ). Similarly, the target trial emulation on 181 patients with SARS-CoV-2 hypoxic pneumonia did not support the effectiveness of the HCQ regimen. ${ }^{65}$

Adding to the controversy, the observational study by Geleris et. al. found no evidence of beneficial or harmful outcomes in the use of HCQ for treating patients with COVID-19. ${ }^{34}$ A separate study by Rosenberg et al. reported that HCQ/AZM treatment was not associated with in-hospital mortality. ${ }^{33}$ The multinational RECOVERY Collaborative Group demonstrated that HCQ administration was not associated with a reduction in 28-day mortality in 4,716 patients. However, there was an increased risk of lengthening the hospital stay, progression to invasive mechanical ventilation or death.

We aimed for this systematic review to help to clear up the controversy surrounding usage of HCQ for COVID-19 treatment. Our meta-analysis found no significant differences in effectiveness of treatment or mortality rates in patients who received either the HCQ or the HCQ plus AZM regimens versus those who were given standard therapy. Furthermore, patients who were given HCQ experienced known adverse effects of HCQ, including vomiting, diarrhoea, blurred vision, rashes, headache, etc.

Interestingly, the findings from our meta-analysis differ from those done by Sarma et al., who analysed 3 studies and concluded that HCQ may have promising effects in the management of COVID-19 patients. ${ }^{13}$ Million et al. ${ }^{12}$ also carried out a meta-analysis on the first available reports on COVID-19 released in IHU Méditerranée Infection. They found a promising trend of beneficial effects of chloroquine derivatives in the treatment of COVID-19, and suggested prescribing HCQ as a Grade I recommendation. Several possible reasons may have contributed to these different conclusions, one of which is that heterogeneity and the pattern of dispersion in the results were not considered by the other researchers. Additionally, the other authors combined treatment outcomes in unusual ways and used odds ratios only in their analysis, whereas risk ratios have higher priority and are the preferred statistic. It is also of concern that non-randomised trials were included in their meta-analyses.

\section{Conclusion}

This systematic review and meta-analysis found no clinical benefits in the use of HCQ, either alone or in combination with AZM, in the treatment of COVID-19. Instead, patients who were given HCQ experienced adverse effects more frequently. It is worth noting that, based on the recommendation of the international steering committee, WHO has discontinued the HCQ and lopinavir/ritonavir treatment arm for the Solidarity Trial on 4 July $2020 .{ }^{66}$ It remains unclear whether hydroxychloroquine is effective for COVID-19 prophylaxis.

\section{Acknowledgments}

The authors would like to thank Dr Alain Rauss for his significant assistance and advice in the clinical and statistical contents of the study, as well as the Student Research Committee of Mazandaran University of Medical Sciences for approving this student research proposal.

\section{REFERENCES}

1. Chew SY, Lee YS, Ghimiray D, et al. Characteristics and Outcomes of COVID-19 Patients with Respiratory Failure Admitted to a "Pandemic Ready" Intensive Care Unit-Lessons from Singapore. Ann Acad Med Singap 2020;49:434-48.

2. World Health Organization. Coronavirus disease (COVID-2019) situation reports. March 2020. Available at: https://www.who.int/ emergencies/diseases/novel-coronavirus-2019/situation-reports. Accessed on 14 November 2020.

3. World Health Organization. Coronavirus disease (COVID-19) advice for the public. 2020. Available at: https:/www.who.int/emergencies/ diseases/novel-coronavirus-2019/advice-for-public . Accessed on 14 November 2020.

4. World Health Organization. COVID 19 candidate treatments; COVID Classification of treatment types. April 2020. Available at: https:/www.who.int/publications/m/item/covid-19-candidatetreatments. Accessed on 14 November 2020.

5. Food and Drug Administration. Coronavirus treatment acceleration program (CTAP). Washington, DC. Available at: https://www.fda.gov/ drugs/coronavirus-covid-19-drugs/coronavirus-treatment-accelerationprogram-ctap. Accessed on 7 October 2020.

6. Pourdowlat G, Panahi P, Pooransari P, et al. Prophylactic recommendation for healthcare workers in COVID-19 pandemic. Adv J Emerg Med 2020;27:e39.

7. Colson P, Rolain JM, Lagier JC, et al. Chloroquine and hydroxychloroquine as available weapons to fight COVID-19. Int J Antimicrob Agents 2020;55:105932.

8. Zhou D, Dai SM, Tong Q. COVID-19: a recommendation to examine the effect of hydroxychloroquine in preventing infection and progression. J Antimicrob Chemother 2020;75:1667-70.

9. Guastalegname M, Vallone A. Could chloroquine/hydroxychloroquine be harmful in Coronavirus Disease 2019 (COVID-19) treatment? Clin Infect Dis 2020;71:888-9.

10. Cavalcanti AB, Zampieri FG, Rosa RG, et al. Hydroxychloroquine with or without Azithromycin in Mild-to-Moderate Covid-19. N Eng1 J Med 2020;NEJMoa2019014.

11. Khuroo MS. Chloroquine and hydroxychloroquine in coronavirus disease 2019 (COVID-19). Facts, fiction and the hype: a critical appraisal. Int J Antimicrob Agents 2020;56:106101. 
12. Million M, Gautret $\mathrm{Ph}$, Raoult D. The efficacy of Chloroquine derivatives in COVID-19: a meta-analysis based on the first available reports. In: Infection IM, editor. IHU Méditerranée Infection. Available at: https://www.mediterranee-infection.com/the-efficacyof-chloroquine-derivatives-in-covid-19-a-meta-analysis-based-on-thefirst-available-reports/. Accessed on 7 October 2020.

13. Sarma P, Kaur H, Kumar H, et al. Virological and Clinical Cure in Covid-19 Patients Treated with Hydroxychloroquine: A Systematic Review and Meta-Analysis. J Med Virol 2020;92:776-85.

14. McGuinness LA. robvis: An $\mathrm{R}$ package and web application for visualising risk-of-bias assessments. National Institute for Health Research (NIHR). 2019.

15. Higgins JPT, Thomas J, Chandler J, et al. Cochrane handbook for systematic reviews of interventions version 6.0. 2nd Ed: John Wiley \& Sons; 2019.

16. Bown MJ, Sutton AJ. Quality Control in Systematic Reviews and Meta-analyses. Eur J Vasc Endovasc Surg 2010;40:669-77.

17. Chen Z, Hu J, Zhang Z, et al. Efficacy of hydroxychloroquine in patients with COVID-19: results of a randomized clinical trial. MedRxiv 2020; doi: https://doi.org/10.1101/2020.03.22.20040758

18. Chen J, Liu D, Liu L, et al. A pilot study of hydroxychloroquine in treatment of patients with moderate COVID-19. J Zhejiang Univ Sci 2020;49:215-9.

19. Mahévas M, Tran VT, Roumier M, et al. Clinical efficacy of hydroxychloroquine in patients with covid-19 pneumonia who require oxygen: observational comparative study using routine care data. BMJ 2020;369:m1844.

20. Tang W, Cao Z, Han M, et al. Hydroxychloroquine in patients with mainly mild to moderate coronavirus disease 2019: open label, randomised controlled trial. BMJ 2020;369:m1849.

21. Gautret P, Lagier JC, Parola P, , et al. Clinical and microbiological effect of a combination of hydroxychloroquine and azithromycin in 80 COVID-19 patients with at least a six-day follow up: an observational study. Travel Med Infect Dis 2020;34:101663.

22. Gautret P, Lagier JC, Parola P, et al. Hydroxychloroquine and azithromycin as a treatment of COVID-19: results of an open-label non-randomized clinical trial. Int J Antimicrob Agents 2020;56:105949.

23. Magagnoli J, Narendran S, Pereira F, et al. Outcomes of Hydroxychloroquine Usage in United States Veterans Hospitalized with COVID-19. Med (N Y). 5 June 2020

24. Molina JM, Delaugerre C, Le Goff J, et al. No evidence of rapid antiviral clearance or clinical benefit with the combination of hydroxychloroquine and azithromycin in patients with severe COVID-19 infection. Med Mal Infect 2020;50:384.

25. Chorin E, Wadhwani L, Magnani S, et al. QT Interval Prolongation and Torsade De Pointes in Patients with COVID-19 treated with Hydroxychloroquine/Azithromycin. Heart Rhythm 2020;17:1425-33.

26. Barbosa J, Kaitis D, Freedman R, et al. Clinical outcomes of hydroxychloroquine in hospitalized patients with COVID-19: a quasi-randomized comparative study. African Society for Laboratory Medicine. Available at: https://aslm.org/resource/clinical-outcomes-ofhydroxychloroquine-in-hospitalized-patients-with-covid-19/.Accessed on 7 October 2020.

27. Million M, Lagier JC, Gautret P, et al. Early treatment of COVID-19 patients with hydroxychloroquine and azithromycin: A retrospective analysis of 1061 cases in Marseille, France. Travel Med Infect Dis $2020 ; 35: 101738$.

28. Yu B, Wang DW, Li C. Hydroxychloroquine application is associated with a decreased mortality in critically ill patients with COVID-19. Sci China Life Sci 2020;63:1515-21.
29. Membrillo de Novales FJ, Ramírez-Olivencia G, Estébanez M, et al. Early Hydroxychloroquine Is Associated with an Increase of Survival in COVID-19 Patients: An Observational Study Preprints 2020; 2020050057.

30. Mallat J, Hamed F, Balkis M, et al. Hydroxychloroquine is associated with slower viral clearance in clinical COVID-19 patients with mild to moderate disease: A retrospective study. medRxiv 2020; doi: https://doi.org/10.1101/2020.04.27.20082180

31. Lee JE, Lee SO, Heo J, et al. Comparative outcomes of lopinavir/ ritonavir and hydroxychloroquine for the treatment of coronavirus disease 2019 with mild to moderate severity. 2020; doi: https://doi.or $\mathrm{g} / 10.1101 / 2020.04 .27 .20082180$

32. Carlucci P, Ahuja T, Petrilli CM, et al. Hydroxychloroquine and azithromycin plus zinc vs hydroxychloroquine and azithromycin alone: outcomes in hospitalized COVID-19 patients. medRxiv 2020; doi: https://doi.org/10.1101/2020.05.02.20080036

33. Rosenberg ES, Dufort EM, Udo T, et al. Association of Treatment With Hydroxychloroquine or Azithromycin With In-Hospital Mortality in Patients With COVID-19 in New York State. JAMA 2020;323:2493-502.

34. Geleris J, Sun Y, Platt J, et al. Observational Study of Hydroxychloroquine in Hospitalized Patients with Covid-19. N Engl J Med 2020;382:2411-8.

35. Arshad S, Kilgore P, Chaudhry ZS, et al. Treatment with hydroxychloroquine, azithromycin, and combination in patients hospitalized with COVID-19. Int J Infect Dis 2020;97:396-403.

36. Chen L, Zhang Z-y, Fu J-g, et al. Efficacy and safety of chloroquine or hydroxychloroquine in moderate type of COVID-19: a prospective open-label randomized controlled study. medRxiv 2020; doi: https:// doi.org/10.1101/2020.06.19.20136093

37. Ip A, Berry DA, Hansen E, et al. Hydroxychloroquine and Tocilizumab Therapy in COVID-19 Patients-An Observational Study. PLoS One 2020; 15:e0237693.

38. Paccoud O, Tubach F, Baptiste A, et al. Compassionate use of hydroxychloroquine in clinical practice for patients with mild to severe Covid-19 in a French university hospital. Clin Infect Dis 2020; ciaa791.

39. Horby P, Mafham M, Linsell L, et al. Effect of Hydroxychloroquine in Hospitalized Patients with COVID-19: Preliminary results from a multi-centre, randomized, controlled trial. medRxiv 2020; doi: https://doi.org/10.1101/2020.07.15.20151852

40. Sbidian E, Josse J, Lemaitre G, et al. Hydroxychloroquine with or without azithromycin and in-hospital mortality or discharge in patients hospitalized for COVID-19 infection: a cohort study of 4,642 in-patients in France. medRxiv 2020; doi: https://doi. org/10.1101/2020.06.16.20132597

41. Castelnuovo AD, Costanzo S, Antinori A, et al. Use of hydroxychloroquine in hospitalised COVID-19 patients is associated with reduced mortality: Findings from the observational multicentre Italian CORIST study. Eur J Intern Med 2020; 10.1016/j. ejim.2020.08.019

42. Albani F, Fusina F, Giovannini A, et al. Impact of Azithromycin and/ or Hydroxychloroquine on Hospital Mortality in COVID-19. J Clin Med 2020;9:2800

43. Lagier J-C, Million M, Gautret P, et al. Outcomes of 3,737 COVID-19 patients treated with hydroxychloroquine/azithromycin and other regimens in Marseille, France: A retrospective analysis. Travel Med Infect Dis 2020;36:101791.

44. Catteau L, Dauby N, Montourcy M, et al. Low-dose hydroxychloroquine therapy and mortality in hospitalised patients 
with COVID-19: a nationwide observational study of 8075 participants. Int J Antimicrob Agents 2020;56:106144.

45. Karolyi M, Pawelka E, Mader T, et al. Hydroxychloroquine versus lopinavir/ritonavir in severe COVID-19 patients. Wien Klin Wochenschr 2020;1-8.

46. Abd-Elsalam S, Esmail ES, Khalaf M, et al. Hydroxychloroquine in the treatment of COVID-19: A multicenter randomized controlled study. Am J Trop Med Hyg 2020;103:1635-9.

47. Peters EJG, Collard D, van Assen S, et al. Outcomes of Persons With COVID-19 in Hospitals With and Without Standard Treatment With (Hydroxy)chloroquine. Clin Microbiol Infect 2020;S1198743X(20)30615-7

48. Singh S, Khan A, Chowdhry $M$, et al. Outcomes of Hydroxychloroquine Treatment Among Hospitalized COVID-19 Patients in the United States - Real-World Evidence From a Federated Electronic Medical Record Network. medRxiv 2020; d oi: https://doi.org/10.1101/2020.05.12.20099028

49. Mitjà $\mathrm{O}$, Corbacho-Monné $\mathrm{M}$, Ubals $\mathrm{M}$, et al. Hydroxychloroquine for Early Treatment of Adults with Mild Covid-19: A RandomizedControlled Trial. Clin Infect Dis 2020; ciaa1009.

50. Regina J, Papadimitriou-Olivgeris M, Burger R, et al. Epidemiology, risk factors and clinical course of SARS-CoV-2 infected patients in a Swiss university hospital: an observational retrospective study. medRxiv 2020; doi: https://doi.org/10.1101/2020.05.11.20097741

51. Okour M, Al-Kofahi M, Austin D. Hydroxychloroquine and azithromycin as potential treatments for COVID-19; clinical status impacts the outcome. J Pharmacokinet Pharmacodyn 2020;47:187-8.

52. Saleh M, Gabriels J, Chang D, et al. The Effect of Chloroquine, Hydroxychloroquine and Azithromycin on the Corrected QT Interval in Patients with SARS-CoV-2 Infection. Circ Arrhythm Electrophysiol 2020;13:e008662.

53. Barbosa Esper R, Souza da Silva R, Costa Oikawa FT, Machado Castro M, Razuk-Filho A, Batista Junior PB, et al. Empirical treatment with hydroxychloroquine and azithromycin for suspected cases of COVID-19 followed-up by telemedicine. Available at: https://pgibertie.files.wordpress.com/2020/04/2020.04.15-journalmanuscript-final.pdf. Accessed on 7 October 2020

54. Ramireddy A, Chugh HS, Reinier K, et al. Experience with Hydroxychloroquine and Azithromycin in the COVID-19 Pandemic: Implications for QT Interval Monitoring. J Am Heart Assoc 2020;9:e017144.

55. Bhattacharya R, Chowdhury S, Mukherjee R, et al. Pre exposure Hydroxychloroquine use is associated with reduced COVID19 risk in healthcare workers - a Retrospective cohort. medRxiv 2020; doi: https://doi.org/10.1101/2020.06.09.20116806

56. Boulware DR, Pullen MF, Bangdiwala AS, et al. A Randomized Trial of Hydroxychloroquine as Postexposure Prophylaxis for Covid-19. N Engl J Med 2020;383:517-25.

57. Mitja O, Ubals M, Corbacho M, et al. A Cluster-Randomized Trial of Hydroxychloroquine as Prevention of Covid-19 Transmission and Disease. medRxiv 2020; doi: https://doio $\mathrm{rg} / 10.1101 / 2020.07 .20 .20157651$

58. Goh KJ, Choong MC, Cheong EH, et al. Rapid Progression to Acute Respiratory Distress Syndrome: Review of Current Understanding of Critical Illness from COVID-19 Infection. Ann Acad Med Singap 2020;49:108-18.

59. Rolain JM, Colson P, Raoult D. Recycling of chloroquine and its hydroxyl analogue to face bacterial, fungal and viral infections in the 21st century. Int J Antimicrob Agents 2007;30:297-308.

60. Detert J, Klaus P, Listing J, et al. Hydroxychloroquine in patients with inflammatory and erosive osteoarthritis of the hands (OA TREAT): study protocol for a randomized controlled trial. Trials 2014;15:412.

61. Yao X, Ye F, Zhang M, et al. In Vitro Antiviral Activity and Projection of Optimized Dosing Design of Hydroxychloroquine for the Treatment of Severe Acute Respiratory Syndrome Coronavirus 2 (SARS-CoV-2). Clin Infect Dis 2020;71:732-9.

62. Pagliano P, Piazza O, De Caro F, et al. Is Hydroxychloroquine a possible post-exposure prophylaxis drug to limit the transmission to health care workers exposed to COVID19? Clin Infect Dis 2020;71:887-8.

63. Roques P, Thiberville SD, et al. Paradoxical Effect of Chloroquine Treatment in Enhancing Chikungunya Virus Infection. Viruses. 2018;10:268.

64. Kim AHJ, Sparks JA, Liew JW, et al. A Rush to Judgment? Rapid Reporting and Dissemination of Results and Its Consequences Regarding the Use of Hydroxychloroquine for COVID-19. Ann Intern Med 2020;172:819-21.

65. Mahevas M, Tran V-T, Roumier M, et al. No evidence of clinical efficacy of hydroxychloroquine in patients hospitalized for COVID-19 infection with oxygen requirement: results of a study using routinely collected data to emulate a target trial. medRxiv 2020; doi: https://doi.org/10.1101/2020.04.10.20060699

66. World Health Organization. WHO discontinues hydroxychloroquine and lopinavir/ritonavir treatment arms for COVID-19. Available at: https://www.who.int/news-room/detail/04-07-2020-who-discontinueshydroxychloroquine-and-lopinavir-ritonavir-treatment-arms-forcovid-19. Accessed on 7 October 2020. 